\title{
GRAIN AND GRAIN BOUNDARY STRUCTURE EVOLUTION WITHOUT TEXTURE CHANGES DURING NORMAL GRAIN GROWTH IN 2-D Al STRIPS
}

\section{SURSAEVA ${ }^{\mathrm{a}}$, U. CZUBAYKO ${ }^{\mathrm{b}, \mathrm{c}, *}$ and A.TOUFLIN ${ }^{\mathrm{a}}$}

a Institute of Solid State Physics, Russian Academy of Science, Chernogolovka, Moscow district, 142432 Russia; ${ }^{b}$ Hahn-Meitner-Institut Berlin GmbH, Glienicker Straße 100, 14109 Berlin, Germany; ' Institut für Metallkunde und Metallphysik, RWTH Aachen, 52056 Aachen, Germany

(Received in final form 28 September 1997)

Changes of the grain boundary character distribution and texture during normal grain growth have been investigated using the SAC-SEM based method and a 4 circle X-ray texture goniometer on Al strips with columnar structure. The microstructure of the strips consists of regions with oriented (clusters) and randomly oriented grains. All changes of microstructure are outside the clusters during normal grain growth and consequently no texture change was observed.

Keywords: Normal grain growth; 2-D grain growth; Grain boundary distribution; Texture; Microstructure evolution

\section{INTRODUCTION}

The process of grain growth is governed by the characteristics, especially grain boundary mobility, of the grain boundaries involved. But many data concerning grain boundary migration obtained by bicrystal experiments and similar methods cannot be adopted perfectly to the understanding of grain growth behaviour in polycrystalline materials. The reason for this is that other factors such as the combination of the

\footnotetext{
* Corresponding author.
} 


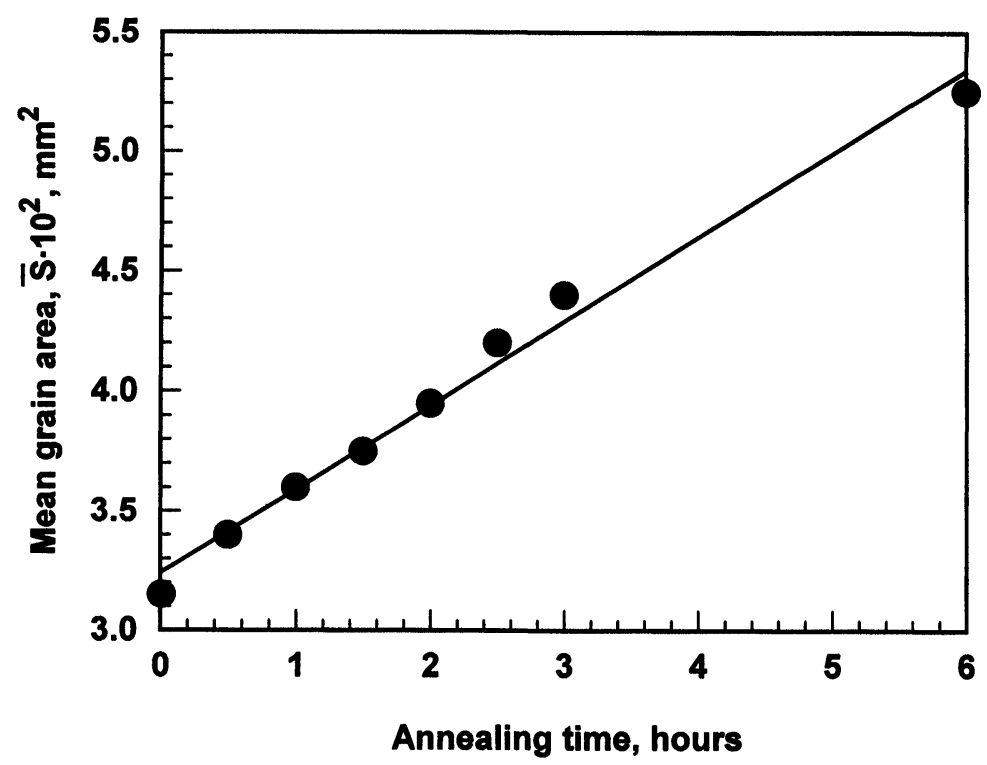

FIGURE 1 The time dependence of the mean grain area. Annealing at $T=773 \mathrm{~K}$. The grain growth data have been collected using linear intercept method for $>500$ grains.

(2) ECP and SEM image were analysed in a complementary way. Orientation, grain area and topological class are measured for 30-40 grains. Moreover the grain boundary length was measured and the axis and angle of misorientation were defined for 60-70 grain boundaries. The grain growth data (grain size and topological class) have been collected using linear intercept method for $>500$ grains. The time dependence of the mean grain area $\bar{S}$ observed in the experiment was determined (Fig. 1). Parallel to the investigation of the microstructure the macrotexture at different annealing times $(0,90,180$ and $360 \mathrm{~min}$ ) were measured in a fully automated 4 circle $\mathrm{X}$-ray goniometer as described by Hirsch et al. (1986). From the obtained four incomplete pole figures the Orientation Distribution Function (ODF), $f(g)$, was computed by the series expansion method (Bunge, 1969).

\section{EXPERIMENTAL RESULTS AND DISCUSSION}

The first part of this chapter is devoted to the evidence that the normal grain growth was observed. Firstly, the evolution of the mean grain 


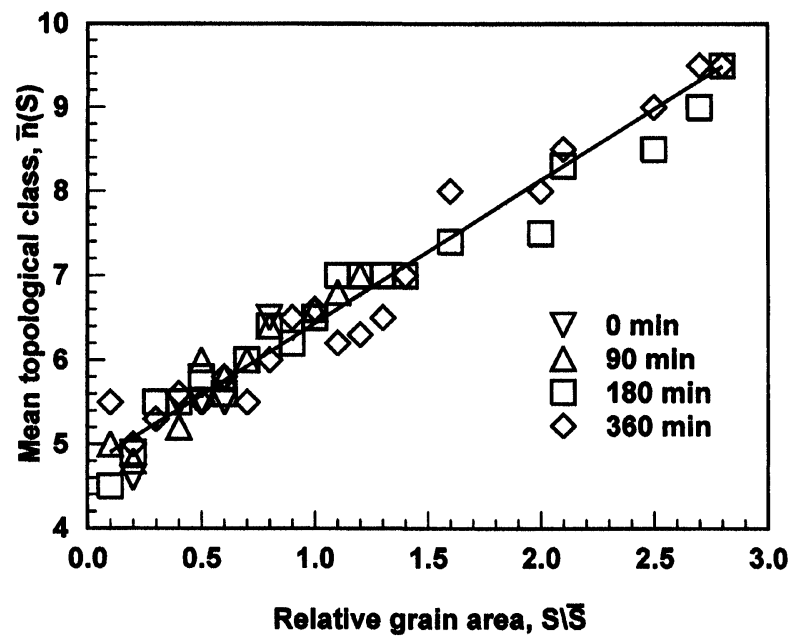

FIGURE 2 The mean topological class vs. relative area of grains.

area $\bar{S}$ depends linearly on the annealing time as shown in Fig. 1. Secondly, the measured distribution of the grain area $S$ follows the well-known exponential law $F(S, t)=\exp (-S / \bar{S}) /(\bar{S})^{2}$. In addition the dependence of mean topological classes $\bar{n}(S)$ on the normalised grain area $S / \bar{S}$ was found to be linear (Fig. 2). Thus the distribution of $S / \bar{S}$ and the distribution of topological classes were steady within experimental error during the entire annealing time. Consequently the microstructure evolution of normal grain growth was observed.

For normal grain growth the grain boundary mobility is determined by the rate of grain growth $\mathrm{d} S / \mathrm{d} t$ (Fig. 1) (Fradkov and Udler, 1989). In the present case the mobility is $A=(3 \pm 0.2) \cdot 10^{-8} \mathrm{~cm}^{2} / \mathrm{s}$. Compared to the present result, the mobility of low-angle tilt grain boundary measured on bicrystals of the same material by Aristov et al. (1981) ranges from $3 \pm 0.2$ to $7 \pm 0.2 \cdot 10^{-8} \mathrm{~cm}^{2} / \mathrm{s}$. So we suppose in this case the kinetic of normal grain growth is governed by the mobility of lowangle grain boundary.

The dependence of the fraction of low-angle grain boundaries on the annealing time was investigated (Fig. 3) also. Shvindlerman et al. (1994) pointed out, that the fraction of low-angle grain boundaries and the area of the corresponding boundaries coincide. As shown in Fig. 3, 


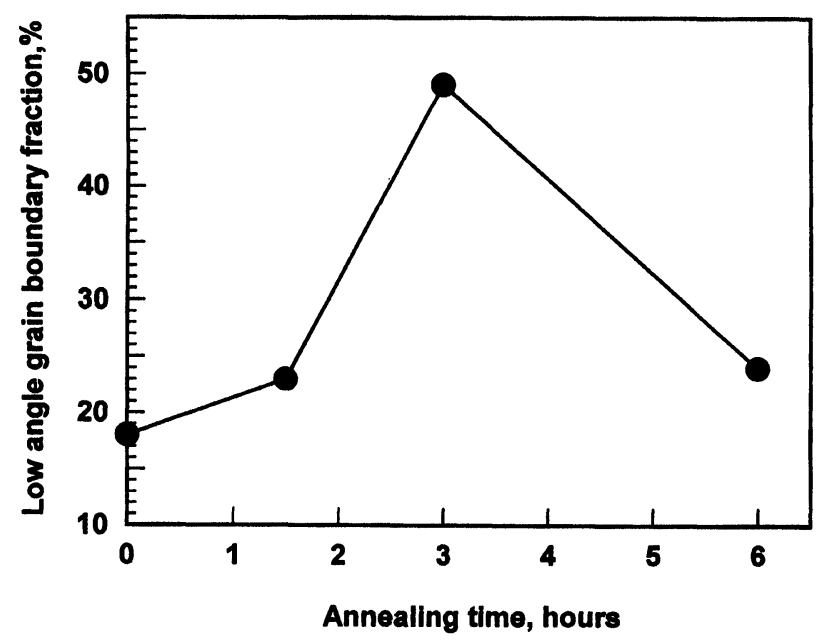

FIGURE 3 The time dependence of the low-angle grain boundary fraction at $T=773 \mathrm{~K}$. The data have been collected for $>200$ grains.

the initial fraction of low-angle grain boundaries is $17 \%$ and reaches a maximum of $50 \%$ at the annealing time of $3 \mathrm{~h}$. At this annealing time the mean grain area is 1.5 times larger than in the initial state (Fig. 4). For larger mean grain area the fraction of low-angle grain boundaries decreases down to approximately $20 \%$. The nonmonotonic time dependence is a result of the competing growth rate of different microstructure components.

The investigation of the microstructure of 2-D aluminium reveals two kinds of regions which are characterised as follows:

(1) Region: In this region the normal direction to the grain surface deviates very little from [100]. The majority of grain boundaries are low-angle boundaries (Fig. 5). A common feature of the experimental results is the tendency of low-angle grain boundaries to occur in clusters. In other words, the low-angle boundaries tend to be associated with small grains.

(2) Region: In this region the grains are oriented randomly. The majority of grain boundaries are large-angle (Fig. 6). We found, that the spatial arrangement of the various types of boundaries is far from random. 


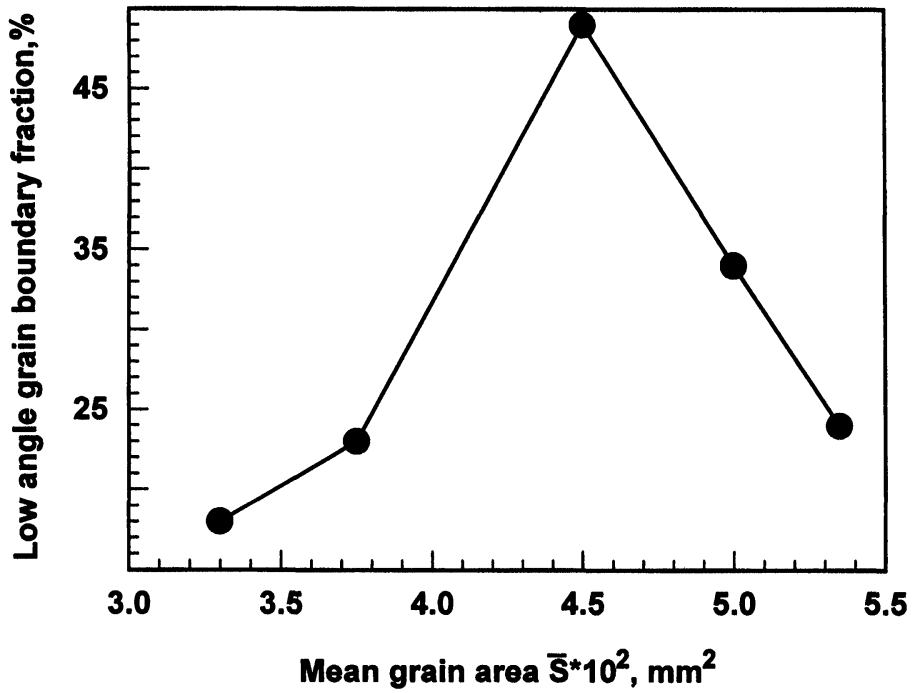

FIGURE 4 The dependence of fraction of low-angle grain boundaries on mean grain area.

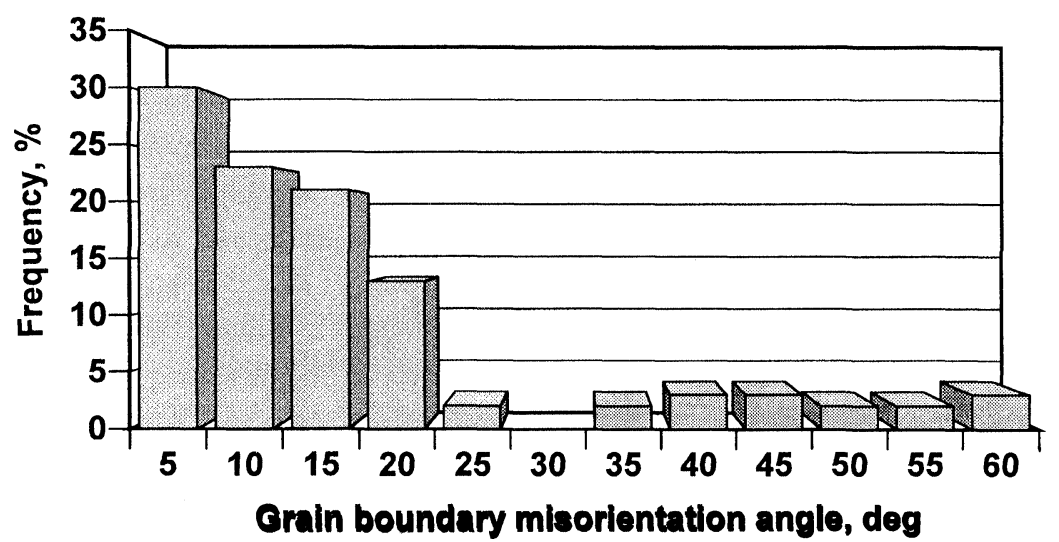

FIGURE 5 The grain boundary frequency distribution vs. the grain boundary misorientation angle for region 1 .

During annealing time the first kind of regions extends at the expense of the second region. Inside the first region there are many low-angle grain boundaries, fewer large-angle grain boundaries and only isolated CSL grain boundaries. Therefore, the important factors in the change of 


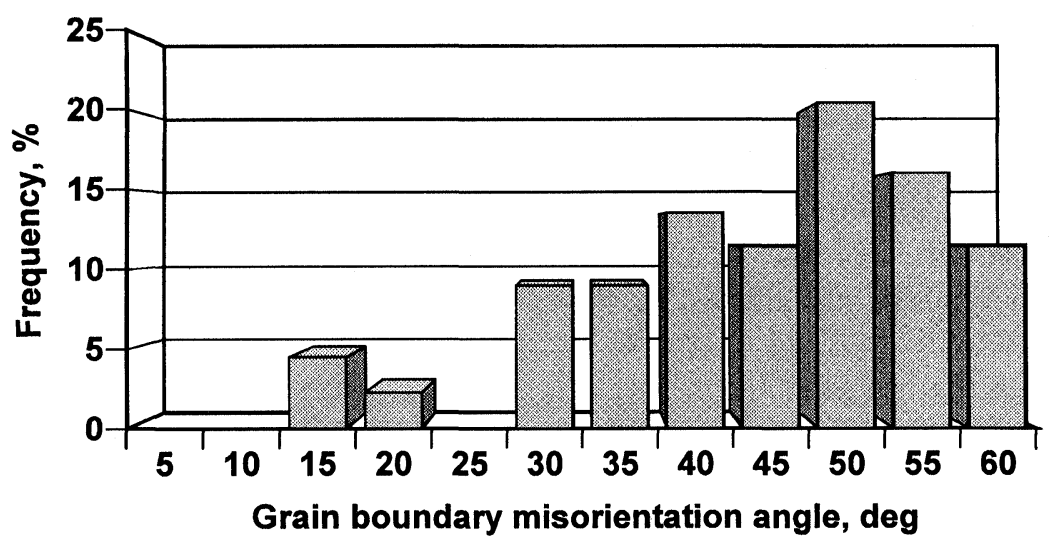

FIGURE 6 The grain boundary frequency distribution vs. the grain boundary misorientation angle for region 2 .

grain boundary character distribution during grain growth are the initial fraction of low-mobility boundaries and the spatial distribution of the characters of grain boundaries. If the fraction of low-angle grain boundaries increases, grain growth can be regarded as an extinction process of grains, which are surrounded by large-angle grain boundaries. Thus, characters of grain boundaries belonging to the disappeared grain and the character of newly formed grain boundary are important in the change of the distribution of the grain boundary characters during grain growth. If the fraction of low-angle grain boundaries decreases, grain growth can be regarded as an evolution of large grains, which are bordered mostly by high angle (random) boundaries with high grain boundary energy. It is likely that low-energy boundaries, which are initially produced for thermodynamic reasons can be modified or replaced by high-energy random boundaries during subsequent grain growth, leading to the reduction of the total grain boundary energy of the system induced by the reduction of the total boundary area. Reduction of the total grain boundary energy of the system does not always imply the reduction of the energy of individual grain boundaries involved in grain growth. In fact, it is possible that the energy of an individual grain boundary is increased during grain growth. This is the case where the rate of change of average boundary energy is smaller than the rate of change of average grain size. 
Parallel to the investigation of microstructure evolution the macrotexture evolution has been studied. Figure 7 shows the $\{111\}$ pole figure of $\mathrm{Al}$ strips in the initial state and annealed at $500^{\circ} \mathrm{C}$ for $360 \mathrm{~min}$, which is representative for all other annealing times. The texture shows a typical recrystallisation texture of $\mathrm{Al}$ with a pronounced cube component $\{001\}\langle 100\rangle$ and a weak $R$ component $\{123\}\langle 634\rangle$. The ODF for each annealing time is presented in Fig. 8 in terms of corresponding skeleton lines and $\beta$-fibres. The $\beta$-fibre is the maximum intensity $f(g)$ for each section $\varphi_{2}=$ const. in Euler space, plotted versus $\varphi_{2}$.

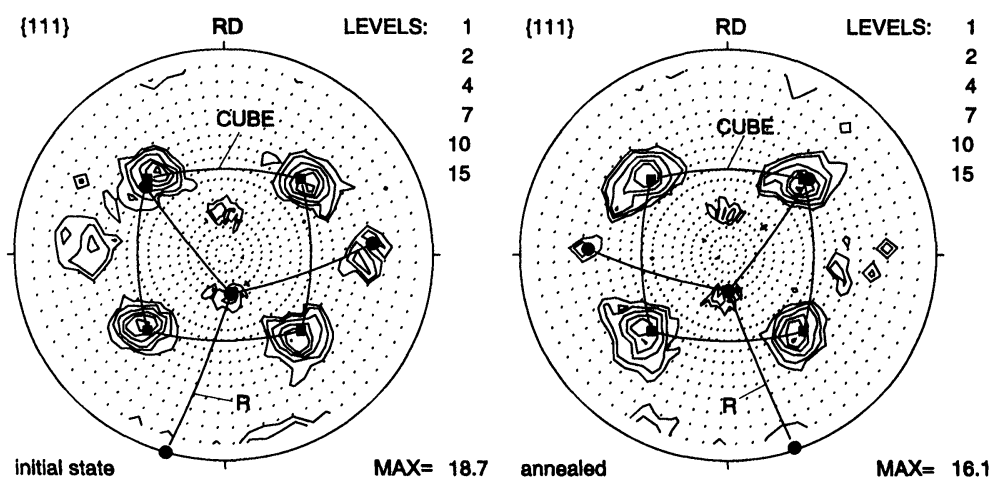

FIGURE $7\{111\}$ pole figures of pure Al strip in the initial state and annealed at $500^{\circ} \mathrm{C}$ for $360 \mathrm{~min}$.

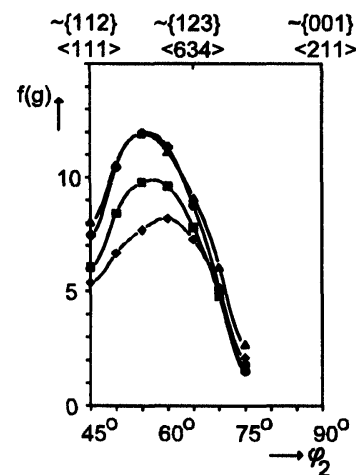

a) $\boldsymbol{\beta}$-fibre (max. density)

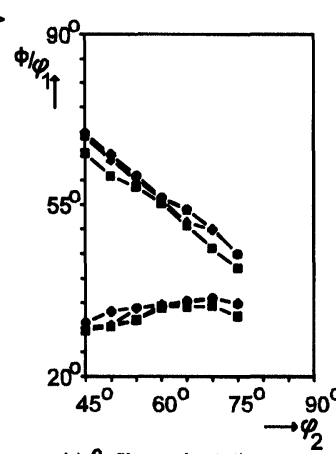

b) $\boldsymbol{\beta}$-fibre orientation

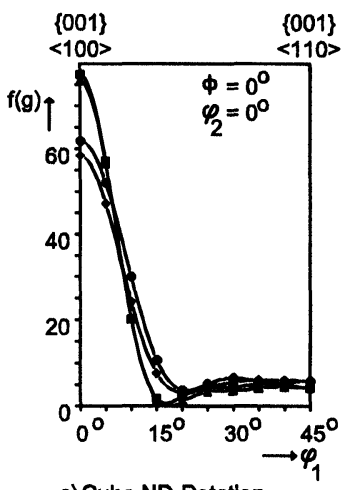

c) Cube ND-Rotation

FIGURE $8 \beta$ fibres (a), (b) and skeleton lines (c) of pure Al strips for different annealing times: $-0 \mathrm{~min},-90 \mathrm{~min},-180 \mathrm{~min}, \boldsymbol{\Delta}-360 \mathrm{~min}$. Annealing temperature $500^{\circ} \mathrm{C}$. 
The strong pronounced cube component (Fig. 8(c)) increases by $25 \%$ for longer annealing times (180 and $360 \mathrm{~min}$ ), whereas the part of the $R$ component (Fig. 8(a)) changes nonmonotonically with increasing annealing time. However, the macrotexture shows only slight texture evolution during the annealing. Thus the change of the low-angle boundary fraction does not affect the macrotexture.

\section{Acknowledgements}

The work was partially supported by the Russian Foundation for Fundamental Research under contract No. 9502054487a and by the Deutsche Forschungsgemeinschaft.

\section{References}

Aristov, V.U., Kopetskii, Ch.V. and Shvindlerman, L.S. (1981). Motion of high-angle tilt grain boundaries in aluminium. In Theoretical Fundamentals of Materials Science, pp. 84-114. Moscow: Nauka.

Bunge, H.J. (1969). Mathematische Methoden der Texturanalyse. Berlin: AkademieVerlag.

Fradkov, V.E. and Udler, D.G. (1989). Normal grain growth in 2-D polycrystals. Pre-print of the Institute of Solid State Physics Russian Academy of Sciences, Chernogolovka.

Hirsch, J., Burmeister, G., Hoenen, L. and Lücke, K. (1986). Autex III - a high speed texture goniometer and data processing system. In Experimental Techniques of Texture Analysis (ed.) H.J. Bunge, pp. 63-71. Oberursel: DGM.

Joy, David, C. (1982). Electron channelling patterns in the scanning electron microscope. J. Appl. Phys., 53, R81-R122.

Shvindlerman, L.S., Sursaeva, V.G., Yashnikov, V.P. and Faulkner, R.G. (1994). Grain boundary misorientation changes during grain growth in pure aluminium. Interface Science, 2, 159-170. 